\title{
Innovación como eje transversal de los modelos de negocio en las organizaciones, una revisión del constructo teórico
}

FECHA DE RECEPCIÓN: 27 de julio

FECHA DE APROBACIÓN: 26 de agosto

pp. 129-148

DOI: http://dx.doi.org/ 10.21158/01208160.n81.2016.1559

\section{Resumen}

En administración y negocios, la innovación se ha posicionado como un elemento diferenciador en las modalidades de negocio al interior de las organizaciones, por ello, este artículo se traza como objetivo establecer una reflexión teórica-conceptual acerca de la innovación en los modelos de negocio. con la finalidad de explorar la relevancia del concepto en la gestión y creación de valor, se contempló una pertinente conceptualización en innovación y modelos de negocio desde una perspectiva interpretativa, así como las ideas y ópticas que destacan entre las formas de innovación y modelos de negocios que crean y generan valor.

\section{Palabras clave}

Gestión de organizaciones, innovación, modelos de negocio, organizaciones.

\section{Luis Fernando Landazury Villalba}

Magíster en Administración de Empresas e Innovación, Universidad Simón Bolívar; especialista en Revisoría Fiscal, Universidad Simón Bolívar y contador público de la misma universidad. Es coordinador de Extensión y Proyección Social de la Facultad de Administración y Negocios, Universidad Simón Bolívar.

\section{Franklin Ferrer Manotas}

Magíster en Administración de Empresas e Innovación, Universidad Simón Bolívar; especialista en Finanzas y Gestión Contable, Universidad Autonóma del Caribe; especialista en Revisoría Fiscal, Universidad Libre y estudiante de la Especialización en Tributación, Universidad Autonóma del Caribe; docente, Corporación Universitaria Americana. 


\title{
Innovation as a Transversal Axis in Organizational Business Models
}

\begin{abstract}
Abstrac. In the field of business and administration, innovation has turned to be a differentiating factor in the business models of organizations; for this reason, this article aims at showing a theoretical reflection about innovation in various business models, exploring the relevance of the concepts of management and creation of added value. It was noticed a pertinent concept for innovation and business models from an interpretation perspective, as well as ideas and views which clearly show that these forms of innovation and business models really generate added value.
\end{abstract}

Key words. Organizational management, innovation, business models, added value, organizations.

\section{L'innovation comme axe transversal des modèles d'affaire des entreprises.}

Resumé. L'innovation étant un élément différenciateur des modalités d'affaire des entreprises, cet article tend à établir une réflexion théorique et conceptuelle de l'impact de l'innovation sur les modalités d'affaire des entreprises. Notre objectif est d'explorer l'importance de ce concept sur la gestion et la création de valeur, de conceptualiser l'innovation des modèles d'affaire à partir d'une perspective interprétative, et de modéliser les types d'innovation et modèles d'affaires créateurs de valeurs.

Mots clefs. Gestion des entreprises, innovation, modalités d'affaire, organisations.

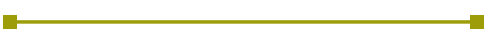

\section{A inovação como eixo transversal dos modelos de negócio nas organizações}

Resumo. Na administração e nos negócios, a inovação tem se posicionado como um elemento diferenciador nas modalidades de negócio no interior das organizações; por isso, este artigo tem como objetivo estabelecer uma reflexão teórico-conceptual sobre a inovação nos modelos de negócio, com a finalidade de explorar a relevância dos conceitos de gestão e criação de valor. Contemplou-se uma conceptualização em inovação e modelos de negócio desde uma perspectiva interpretativa, assim como as ideais e visões que se destacam entre as formas de inovação e modelos de negócios que geram e criam valor.

Palabras chave. Gestão de organizações, inovação, modelos de negócio, organizações. 


\section{Introducción}

T $\mathrm{n}$ el contexto mundial, el fenómeno de $\checkmark$ la globalización ha generado a nivel microeconómico un constante cambio, evolución y reestructuración de las estrategias utilizadas por las empresas (Stiglitz, 2002). De igual forma, teorías referentes al desarrollo endógeno han abierto camino a nuevas metodologías y formas mas organizacionales cuyo factor motriz se ubica en la investigación, desarrollo e innovación (Amar y Diazgranados, 2006). Dichos cambios han sido dinámicos e incrementales en lo referente a la expectativa de los mercados, y es aquí donde radica el éxito de diferentes organizaciones, que han sido capaces de agregar valor a sus productos o servicios para satisfacer los requerimientos de los clientes y ser competitivas en el mercado.

El mercado colombiano no se escapa de esta dinámica y cada día se torna más exigente, generando expectativas muy altas de parte de los clientes; sin embargo, el sistema gubernamental de la nación poco ha invertido para potencializar y producir en las organizaciones sistemas de gestión de investigación, desarrollo e innovación que permitan fomentar la creación y desarrollo de nuevos o mejores productos, procesos y modelos de negocio, que satisfagan y se adapten a las nuevas necesidades del mercado (Montoya, A.; Montoya, I. y Castellanos, 2010).

En este rango de ideas, estos autores manifiestan que la mayoría de las empresas colombianas desconocen los métodos para mejorar los procesos, servicios y agregar valor a los productos, por tanto, su área de operación está siempre inscrita en la geografía nacional, desaprovechando, en muchos casos, su verdadero potencial, recursos y capacidades competitivas. Este rezago en el ascenso de la innovación hacia un lugar privilegiado dentro de la organización, también puede ser visto a nivel nacional, pues aún hoy sigue en construcción una cultura de la innovación, dado que la relación entre los agentes que componen el sistema de innovación, universidad-empresa-Estado, sigue siendo precaria (Mendoza, 2000). De acuerdo con Amar, Angulo, Ortega y Quintero (2006), existe un conjunto de metodologías y teorías que orientan la integración de estos elementos, como el triángulo de Sábato (Sábato y Botana, 1968), el modelo de cuatro entornos (Fernández de Lucio y Conesa, 1996) y el modelo de triple hélice (Etzkowitz y Leydesdorff, 2000), entre otros, principios que sustentan el esfuerzo de

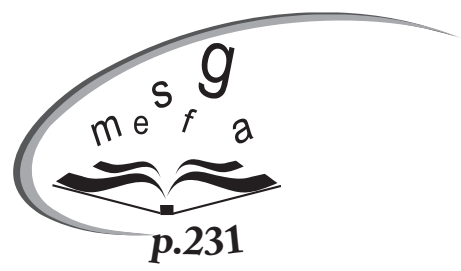
la comunidad científica por establecer un marco de referencia para la interrelación de los agentes en el desarrollo científico y tecnológico de los territorios y las organizaciones.

Hoy en día, las organizaciones también deben poder adaptarse con rapidez y efectividad para sobrevivir ante panoramas basados en la incertidumbre y el caos, pues la velocidad y complejidad del cambio en el entorno, a menudo ponen a prueba las capacidades de los sistemas empresariales por mantener estructuras organizacionales flexibles que permitan adecuarse de manera rápida a los cambios del entorno (Peña, Soria y Velázquez, 2012).

Uno de los aspectos relevantes que permiten a las organizaciones ser competitivas en mercados tan turbulentos, como lo señala Porter (2009), es la innovación, y una de sus manifestaciones en las empresas es la innovación organizacional, la cual lleva inmerso 
un proceso de transformación interna sobre toda la cultura empresarial para impulsar el crecimiento de una industria capaz de competir con mayor oportunidad y herramientas tanto en el escenario nacional como internacional, razones por las cuales se sustenta la relevancia del desarrollo de la investigación.

En concreto, luego de que una empresa implementa el modelo gerencial acorde con sus características que le producen ventajas competitivas, se convierte en referente para otras empresas, lo que genera un cúmulo de lecciones aprendidas que despierta, bien sea dentro de su mismo sector o en uno diferente, el interés por construir modelos propios de gestión basados en la innovación, para obtener, de esta manera, los beneficios que se logran al consolidar un entorno que impulsa simultáneamente la innovación dentro de la compañía y en el sector productivo en que se encuentre; este hecho que Clusters altamente innovadores y robustos.

Se hace relevante estudiar los modelos organizacionales innovadores como una estrategia capaz de generar ventaja competitiva, teniendo en cuenta que este tipo de modelos llevan implícito un cambio en la forma de pensar de las personas en la organización, lo cual propicia y estimula una cultura organizacional altamente participativa en todas las áreas de la empresa y durante todas las etapas de los proyectos que se desarrollan. Esto, permite entrever que solo por este mecanismo, el conocimiento concentrado en el talento humano pueda liberarse y direccionarse para lograr una mayor ventaja y eficiencia en las organizaciones.

En el presente artículo se exploran dos principales vertientes desde una perspectiva conceptual: en primer lugar, los modelos de negocio que analizan la forma estructural de las organizaciones, y la modalidad de creación y circulación del valor, así como los elementos que participan en esta actividad. Este enfoque permite contemplar la segunda vertiente, la innovación, como una estrategia integradora y generadora de cambio ubicada en la instancia de un elemento transversal que aporta a la diferenciación de la organización; en la misma medida, se analizan modelos puntuales que diversos referentes han desarrollado sobre este concepto. De esta forma, se contrastan las diferentes teorías, y mediante la óptica crítica se generan las discusiones finales y las conclusiones que demarcan la relación entre la creación del modelo de negocio en las organizaciones y la integración de la innovación como respuesta a la diferenciación en el contexto. 


\section{Modelos de gestión y negocios}

$\mathrm{D}^{\mathrm{e}}$

e acuerdo con Álvarez (2009), el modelo de gestión es una referencia o guía de administración de una empresa. Sobre este modelo, se basan las acciones que se toman ante una situación determinada para alcanzar los objetivos de la empresa. En este sentido, el modelo de gestión engloba el componente de gobernanza, tanto de las políticas como de los activos que se exponen en el modelo de negocio.

Así mismo, Tejada (2003), manifiesta que son indisolubles los vínculos entre la gestión del talento y la gestión del conocimiento, porque por medio de dicha unión, se gestan cuatro características gerenciales, que se erigen como patrones básicos que un sistema gerencial debe desarrollar para que el proceso de administración sea exitoso. Menciona el reconocimiento de sí mismo, y a partir de esto, constituirse en autotransformador del cambio. Se trata de un parámetro muy importante, sobre todo en empresas que han reconocido la innovación como el soporte de su ventaja competitiva; muchas veces el miedo a cometer errores inhibe la implementación de nuevas ideas.

Lo anterior pone de manifiesto que no es suficiente con el hecho de que un determinado grupo o división de la organización tenga los recursos financieros y técnicos que requiere un ambiente para la innovación, sino que de manera fundamental debe existir un talento humano que se autorreconoce como transformador del cambio. Otro componente, ahora del lado de la organización, manifiesta que se debe también procurar por animar a las personas a ser creativas y perder el temor a cometer errores, por medio de capacitaciones y desarrollo de talleres grupales. En todo esto, los incentivos desempeñan un papel muy importante, puesto que al final del proceso creativo el talento se dará cuenta de que es capaz de implementar nuevas ideas.

Tejada (2003), como segundo patrón, menciona el reconocimiento del otro, y a partir de esto, el constituirse en facilitador del cambio del otro. Adicionalmente, sugiere, como tercer patrón, el reconocimiento del entorno o contexto y el constituirse en agente de su transformación; finalmente, establece el desarrollo de competencias de alta calidad y actualidad.

En este sentido, Tejada (2003), reconoce que el gerente debe construir los procesos de cambio a partir del desarrollo de estrategias que permitan dilucidar fortalezas y debilidades, tanto propias como de sus colaboradores; de igual modo, direcciona las transformaciones a través de: liderazgo, cultura, tecnología, sistemas de medición y política de recursos humanos, que actúan como facilitadores del flujo del conocimiento (Revilla, 1999).

Entre los modelos de gestión basados en el conocimiento, es importante resaltar que dicho principio debe procurarle a la organización la adquisición de patrones de acción y fortalecer los comportamientos adquiridos, así como crear y almacenar conocimiento para su uso, que permita la generación de valor y ventajas competitivas a la empresa. Lo anterior hace pensar la necesidad de integrar los modelos de gestión de conocimiento a la política del recurso humano de toda organización que desarrolle procesos paralelos de formación, instrucción, acompañamiento, redireccionamiento y seguimiento a la capacidad de generar conocimiento a la empresa como factor diferenciador en el mercado (Revilla, 1999). 
Pese a la amplia utilización del concepto modelo de negocio, no existe una definición genérica y aceptada que englobe lo que realmente significa, además, no es solo lo que pobremente citan algunos autores con respecto a la manera en la que opera la compañía (Casadesus-Masanell y Ricart, 2007).

Amit y Zott (2001), mencionan que «un modelo de negocio describe el contenido, la estructura y gobernancia de las transacciones diseñadas para crear valor a través de la explotación de las oportunidades de negocios». En esta definición, la transacción se refiere a los bienes o información que está siendo intercambiada, tanto como los recursos y capacidades requeridas para ellos. La estructura de la transacción se refiere a las partes que participan, sus redes y la forma en la que eligen operar. Finalmente, la gobernancia hace referencia a la manera como fluye la información, los recursos y los bienes que son controlados por las partes, el marco institucional y los incentivos que tienen los participantes.

Como se puede apreciar, esta definición toma apuntes de diferentes teorías, que por sí solas, no explican, en consideración de los autores, lo que realmente significa un modelo de negocio. Dicha definición toma nota de las teorías de los mercados virtuales y del emprendimiento, que desde el punto de vista de Schumpeter (1989), implica análisis de cadenas de valor, punto de vista de los recursos, capacidades dinámicas, teoría de los costos de transacción y redes estratégicas.

Teniendo en cuenta los aportes de Drucker (2007), Amit y Zott (2001), Magretta (2002), Casadesus-Masanell y Ricart (2007), quienes definen al modelo de negocio como el conjunto de elecciones que realiza la empresa y sus consecuencias, las elecciones pueden ser del orden de la política de funcionamiento, activos físicos en los que invierte, y las estructuras de gobierno o modelo de gestión que utiliza para implantar las políticas y gobernar los activos. Este sistema de elecciones configura la forma de operación de la empresa.

Un componente importante de los modelos de negocio son las decisiones específicas realizadas por la gerencia sobre cómo debe operar la organización. Por ejemplo, las decisiones sobre las prácticas de compensación, contratos de cumplimiento, ubicación de las instalaciones, es decir, la manera como la compañía operará en el tiempo.

Sin embargo, las decisiones, por sí solas, no constituyen un modelo de negocio. Estas deben estar integradas a los procesos de creación de valor y de captura del mismo valor. Y por tanto, también debe estar integrado a las decisiones, el conjunto de consecuencias que conlleva tomar ciertas determinaciones. Por ejemplo, decisiones sobre las políticas de precios tienen implicaciones sobre el volumen de venta, y esto, a su vez, afecta la escala de economías y el poder de negociación de la compañía (Casadesus-Masanell y Ricart, 2007).

Las políticas hacen referencia a las acciones adoptadas por la compañía, que tienen que ver con todos los aspectos de la operación. Por ejemplo, una política podrá ser la ubicación de la planta de una empresa en un área rural o brindar a los trabajadores incentivos monetarios. Los activos físicos tienen en cuenta todos los recursos, como las instalaciones de manufactura o infraestructura, y de comunicación entre las oficinas; la gobernanza se refiere a la estructura de arreglos contractuales que otorgan derechos sobre las políticas y activos. 
Dicho norte o estrategia para atraer a los consumidores de la propuesta de valor, lleva implícita la idea de innovación, pues una empresa que no integra el concepto de innovación en su estrategia o modelo de negocio, cederá con el tiempo su cuota de mercado al momento de competir con empresas que si lo hacen.

Por otra parte, Álvarez (2009, p. 15), propone que un modelo de negocio «es una planificación que realiza la empresa de sus ingresos y beneficios futuros, que establece el norte a seguir para atraer a los clientes, definir ofertas de productos e implementar estrategias para tener éxito en el mercado».

De igual forma, Casadesus-Masanell y Ricart (2007), plantean que la innovación es un factor que en los modelos de negocio se está convirtiendo en una de las fuerzas principales que direccionan los esfuerzos de renovar la estrategia de los negocios en el mundo.

En este punto, en el que se concibe al modelo de negocio como estrategia ante la innovación (Tabla 1), es bueno resaltar los aportes que realizaron Freeman (1975) y Formichella (2005).

Tabla 1. Modelo de negocio como estrategia ante la innovación.

\begin{tabular}{|c|c|}
\hline Estrategia & Definición \\
\hline $\begin{array}{c}\text { Estrategia innovadora } \\
\text { ofensiva }\end{array}$ & $\begin{array}{l}\text { Es aquella que pretende conseguir el liderazgo técnico y de mercado } \\
\text { posesionándose, primero, frente a sus competidoras y llevando la } \\
\text { delantera en la introducción de nuevos productos o procesos; estas } \\
\text { empresas se caracterizan por su intensiva gestión de investigación. }\end{array}$ \\
\hline $\begin{array}{c}\text { Estrategia innovadora } \\
\text { defensiva }\end{array}$ & $\begin{array}{l}\text { La diferencia con la estrategia ofensiva está en la naturaleza y en } \\
\text { el ritmo de las innovaciones. El innovador defensivo no tiene como } \\
\text { objetivo ser el primero, pero tampoco quiere quedarse atrás del } \\
\text { cambio. }\end{array}$ \\
\hline Estrategia i & $\begin{array}{l}\text { La firma imitativa no tiene como objetivo pasar a las demás, ni } \\
\text { siquiera mantenerse en el juego. Se conforma con marchar detrás } \\
\text { de las líderes. Su estrategia es dependient y este tipo de firmas no } \\
\text { intenta iniciar o incluso imitar cambios técnicos en su producto. }\end{array}$ \\
\hline Estrategia tradicional & $\begin{array}{l}\text { La firma tradicional se diferencia de la dependiente en que su } \\
\text { producto cambia poco o no cambia nada. El producto de la firma } \\
\text { dependiente puede cambiar muchísimo, pero siempre en respuesta } \\
\text { a una iniciativa y una especificación desde afuera. }\end{array}$ \\
\hline $\begin{array}{c}\text { Estrategia oportunista } \\
\text { o de nicho }\end{array}$ & $\begin{array}{l}\text { Existe siempre que un empresario detecte alguna oportunidad } \\
\text { en un mercado rápidamente cambiante, que le permite encon- } \\
\text { trar un nicho importante y ofrecer un servicio o producto que } \\
\text { los consumidores necesitan, pero que nadie más pensó en } \\
\text { suministrarlo. }\end{array}$ \\
\hline
\end{tabular}

Fuente. Elaboración propia a partir de Formichella (2005). 
Vale la pena resaltar el hecho de que la innovación constituye un importante elemento del quehacer de las organizaciones, dado que se ubica como una fuente de generación de valor en el contexto de las empresas (Hamel, 2006). En la práctica de los negocios, este factor suele confundirse con la invención y la generación de nuevos productos; si bien este paradigma está relacionado, la innovación verdadera visiona más allá del simple hecho de diseñar nuevos productos, ya que las propias empresas se ven inmersas en el contexto de crear formas distintas de hacer negocios para ser competitivas, fundamentándose en competencias distintivas, como el servicio al cliente, la calidad, los cuadros de mando, la misma innovación, entre otras; este es un principio que ha formado parte del pensamiento administrativo (Melamed, 2015). En este orden de ideas, en el ejercicio de la innovación, en este caso del modelo de negocio, Johnson, Christensen y Kagermann (2008) manifiestan que un modelo de negocio es la modalidad con la cual una empresa se esfuerza por la creación, el desarrollo y distribución de valor; esta idea es complementada por Osterwalder y Pigneur (2013), quienes resaltan la ferviente necesidad de las empresas por generar ingresos para ser rentables y sostenibles, ofreciendo valor a su segmento de clientes, valor que se manifiesta en rentabilidad para la arquitectura de la firma, una red de beneficios para sus grupos de interés en el entorno y capital relacional para sus clientes finales.

El ambiente de los negocios exige a las organizaciones, independientemente de su actividad económica, formular objetivos y desarrollar estrategias que propicien ser sostenibles en el corto, mediano y largo plazo. Dadas las cambiantes condiciones del entorno, esta actividad representa un reto, no obstante, la principal idea de las organizaciones se orienta a la relación con el ambiente y a la generación de valor con sus grupos de interés (Zott y Amit, 2005). En este sentido, es importante enunciar que la metodología por la cual una empresa genera valor es conocida como modelo de negocio. Osterwalder (citado en Márquez, 2010, p. 31), propone una definición referente a los modelos de negocio afirmando que:

Un modelo de negocio es una herramienta conceptual que, mediante un conjunto de elementos y sus relaciones, permite expresar la lógica mediante la cual una compañía intenta ganar dinero generando y ofreciendo valor a uno o varios segmentos de clientes, la arquitectura de la firma, su red de aliados para crear, mercadear y entregar este valor, y el capital relacional para generar fuentes de ingresos rentables y sostenibles.

Al analizar el referente de Osterwalder (2007), en contraste con la afirmación de Johnson, Christensen y Kagermann (2008), quienes manifiestan que un modelo de negocio es la interrelación de diferentes elementos que articulados crean y desarrollan valor, el común denominador entre estas definiciones se orienta a la interacción de diferentes componentes de las organizaciones en torno a sus grupos de interés y a la generación de valor. Cabe destacar, que desarrollar un modelo de negocio, según Dorf y Blank (2012), no implica la formación de un startup ${ }^{1}$, realmente, la exploración de una fórmula generadora de valor requiere lineamientos, estrategias, competencias y herramientas diferenciadoras

\footnotetext{
${ }^{1}$ Un startup es la versión más pequeña de una gran compañía (Dorf, 2015).
} 
que faciliten la optimización del modelo, minimizando el riesgo y fortaleciendo las oportunidades de éxito. Entonces, es importante tener en cuenta qué elementos forman parte de los modelos de negocio, no obstante, estos también pueden concebirse como la abstracción de la realidad a una idea que retrata la radiografía de funcionamiento actual de la actividad; es donde se asume la óptica global de los modelos de negocio que generalmente transitan por una serie de procesos de declaración, análisis, ejecución, control y compensación, por ello, deben alinearse a la dirección estratégica de las organizaciones buscando la perfecta sinergia entre el desarrollo competitivo y la generación de valor (Jofré, 2002).

La arquitectura de un modelo de negocio exitoso, de acuerdo con Johnson, Christensen y Kagermann (2008), debe someterse a un continuo proceso de reinvención, dadas las contingentes condiciones del entorno. Sin embargo, y por lo general un modelo de negocio articula cuatro elementos principales: la propuesta de valor, la fórmula de beneficios, las actividades y recursos clave. Cabe agregar, que, de acuerdo con Márquez (2010), en el diseño de los modelos de negocio la innovación debe ser eje transversal implícito que impacte sobre los demás elementos, de esta forma, se genera el componente diferenciador que potencializa el éxito del modelo; así como contemplar una red de intercambio de factores que se potencien a través del cambio y permitan el debido posicionamiento en las escalas del mercado (Turner y DeVaughn, 2008).

Esta serie de elementos clave representan la base para la generación de metodologías más concretas en términos de diseño de modelos de negocio. Osterwalder y Pigneur (2013), son pioneros en una metodología frecuentemente empleada en la formulación de planes de negocio, denominada Canvas. Esta interesante herramienta consiste en la organización simple y sistemática de los elementos -actividades, recursos y aliados claves, propuesta de valor, medios de distribución, segmentos de mercado, relaciones con el cliente, costos y fuentes de ingreso-, que giran en torno a la generación de valor (López, 2014). La metodología Canvas se sustenta en la idea de integrar elementos categorizados en cinco pilares: lienzo -despliegue de elementos del modelo de negocio-; patrones -relación entre factores-; diseño -enfoque y orientación del negocio-; estrategias -plan de acción, respuestas y contingencias-; y procesos -programación de actividades-, que conforman la estructura del modelo de negocio (Osterwalder y Pigneur, 2013).

Al analizar los distintos referentes de los elementos que integran un modelo de negocio, no se difiere en el término de la caracterización en torno a los recursos incidentes en el valor que se genera y canaliza en las organizaciones; es donde surge el proceso de diseño de un modelo de negocio que se oriente a una visualización, evaluación, planeación, comunicación e implementación del modelo en los diferentes niveles de la organización, agregando como un halo transversal a la innovación, elemento que fortifica la capacidad creativa, de cambio y la distinción de la abstracción del modelo de negocio (Márquez, 2010).

Estas definiciones no ubican en su marco de referencia a la innovación, no obstante, Jofré (2002), manifiesta que este componente es de gran relevancia y que su existencia en las organizaciones debe ser implícita en las operaciones diarias. Así mismo, Márquez (2010), sustenta esta idea afirmando que la innovación es una idea transversal en las 
diferentes dependencias de la estructura organizacional; ahora existen de igual forma una serie de postulados que soportan la arquitectura de un modelo de negocio. Johnson, Christensen y Kagermann (2008), expresan que existen cuatro principales componentes de un modelo de negocio exitoso:

- Propuesta de valor ofrecida al cliente. En primera instancia, un modelo de negocio exitoso conoce y delimita el segmento del mercado al que apunta; posterior a este proceso de caracterización, es donde se fragua la oferta de producto revestida de las competencias distintivas de la organización, que hacen de garante de la competencia con diferenciación de los competidores directos e indirectos.

- Fórmula de beneficios. Los modelos de negocio de éxito no desconocen que la meta de las empresas es generar dinero (Goldratt y Cox, 2010); por ello, también deben estructurarse las dimensiones que crean y generan renta para la empresa, como lo son los volúmenes de venta, los ingresos, costos, erogaciones y la capitalización (Stettenius, Doyle y Colley, 2009).
Esta serie de aspectos financieros siempre deben apuntar a la creación de beneficios para la empresa.

- Recursos clave. Las organizaciones son, en esencia, un conjunto de recursos que integrados coadyuvan al logro de una serie de objetivos en el tiempo, por ello, es importante identificar y fortificar aquellas personas, tecnología, información o medios que faciliten el desarrollo del negocio.

- Actividades clave. Al igual que los recursos, las políticas, reglamentos, normas, procedimientos y actividades que contribuyan en mejor manera al desarrollo del negocio, son los lineamientos claves en la estructura de éxito del modelo de negocio.

Cabe destacar que Johnson, Christensen y Kagermann (2008), también manifiestan que los modelos de negocio se ajustan a las condiciones del entorno, por lo que deben siempre estar ondeando en un círculo continuo de mejoramiento, pues en este proceso de reinvención es donde se evidencia el componente innovador que fortalece la capacidad diferenciadora del negocio en el mercado. 


\section{Gestión de la innovación}

E $\mathrm{n}$ las primeras décadas del siglo $\mathrm{XX}$, Schumpeter (2010), introdujo, por primera vez, el término innovación como eje del desarrollo del capitalismo y del mejoramiento de la distribución global del ingreso y la producción. Posteriormente, han sido muchos los autores que han interpretado el concepto de innovación según las necesidades económicas reinantes de la época debido a su constante evolución y complejidad.

Entre los autores que se destacan por haber conceptualizado sobre las teorías tecnológicas y de innovación, están: Zaltman, Duncan y Holbeck (1973); Gee (1981), Freeman (1975), Foster (1987) y Porter (1991), en la década de los 90 y principios del siglo XXI. Además, cabe destacar el aporte significativo que ha dado la Organización de Cooperación y Desarrollo Económico (OCDE, 2005) a la evolución y el manejo de los conceptos relacionados con la ciencia, la tecnología y la innovación.

En este sentido, la importancia del paradigma tecnológico ha conllevado a la aparición de disciplinas teóricas y prácticas que permitan encauzar de manera eficiente dicho desarrollo, de tal manera que la apropiación de la tecnología en las organizaciones incorpore inteligencia a los negocios, soportando la toma de decisiones (Azoumana, 2013). Unas de las prácticas en las organizaciones ha sido la gestión de la tecnología y la gestión de la innovación. Esta metodología aparece como necesidad de los sistemas organizativos para liderar sus procesos tecnológicos en aras de una mayor competitividad (Mendoza, 2000).

Los planteamientos de Wilmot y Carlson (2006), y Porter (2001), afirman que la innovación es entendida como el proceso consciente de transformación de ideas para la creación de valor. Dicho proceso inicia con la concepción común de los individuos encargados de la dirección estratégica de la empresa u organización interesada en mantener e incrementar su participación en un mercado específico.

Para Mendoza (2005), las organizaciones están inmersas en una fuerte competencia en los mercados lo que ha conllevado a que estas realicen grandes esfuerzos para la generación de valor, la reducción de los costos y el encadenamiento con sus grupos de interés que permitan crear ventajas competitivas. De allí, que las compañías se esmeren por crear nuevas formas de desarrollo empresarial.

Precisamente, el reto más importante del proceso de innovación es que la comunidad, en su totalidad, comprenda la importancia de mirar más allá del éxito relativo que tienen el producto o servicio a lo largo del tiempo, y se conciba, en su lugar, un modelo dinámico de cambio que vaya de acuerdo con las nuevas expectativas y exigencias de los consumidores.

No obstante, el resto de los eslabones de la cadena de innovación no son menos difíciles, pues todavía están pendientes definiciones y procedimientos concretos para la medición, gestión y evaluación por medio de indicadores, la actividad innovadora dentro de la organización.

El proceso creativo de generar ideas y transformarlas posteriormente en prototipos, productos comercializables, y finalmente, constitución de empresas, ha tenido a lo largo de los años una interpretación así (Mendoza, 2000). Inicialmente, Schumpeter (1989), concibe la actividad de innovar como la realización de 
nuevas combinaciones -recombinaciones de elementos materiales y cognitivos que anteriormente existían-. Son los autores Wilmot y Carlson (2006), quienes complementan la idea anterior abriendo paso, no solo a la mera combinación, sino a la implementación de ideas que generan valor para el consumidor, y crecimiento económico para las empresas.

La OCDE (2005), establece que la innovación es la introducción de un nuevo, o significativamente mejorado, producto - bien o servicio-, de un proceso, de un nuevo método de comercialización o de un nuevo método organizativo, en las prácticas internas de la empresa, la organización del lugar de trabajo - las relaciones exteriores. Una empre-sa innovadora, en cuanto a producto/proceso, es la que ha introducido un nuevo producto o proceso, o lo ha mejorado significativamente, durante el periodo en estudio.

Así mismo, la OCDE (2005), también define en su Manual de Oslo cuatro tipos de innovación según su naturaleza: innovación de productos, innovación de procesos, innovación organizativa e innovación en mercadotecnia (Tabla 2).

Tabla 2. Tipos de innovación.

\begin{tabular}{|l|l|}
\hline \multicolumn{1}{|c|}{ TIPO } & \multicolumn{1}{c|}{ CONCEPTO } \\
\hline Innovación de producto. & $\begin{array}{l}\text { Su mayor diferencia radica en la novedad de presentación, morfología } \\
\text { o composición. }\end{array}$ \\
\hline Innovación de proceso. & $\begin{array}{l}\text { Es la adopción de métodos de producción tecnológicamente nuevos } \\
\text { o sensiblemente mejorados. }\end{array}$ \\
\hline Innovación organizativa. & Se refiere a la puesta en práctica de nuevos métodos de organización. \\
\hline Innovación en mercadotecnia. & Implican la puesta en marcha de nuevos métodos de comercialización. \\
\hline
\end{tabular}

Fuente. Ortiz (2014).

Hay que señalar, tal como lo plantea Ortiz (2014, p. 80), "que entre la innovación de producto y la innovación de procesos existe más que diferencias es más sinergia entre ellas y en su conjunto se le conoce como innovación tecnológica».

Según Porter (1990), en una sola oración resume todo lo anterior, y abre paso a lo que actualmente se entiende como la definición de la innovación: «innovar es crear valor nuevo y significativo para el mercado». Porter (citado en Del Campo, 2009, p. 24), va más allá al asegurar que «las empresas más exitosas han llegado a serlo por su capacidad para innovar en la creación de valor para sus consumidores», y anota en su cita de manera concreta, que «la única ventaja competitiva sostenible es la innovación permanente».

La creación de valor, como se ha podido notar, es un concepto general que no solo está relacionada con aspectos tecnológicos, aun cuando van muy de la mano. Complementa la idea y abarca otros escenarios dentro de la organización, como aquellos relacionados con la estrategia de mercadeo, modelo organizacional, práctica de negocios y aquellas áreas funcionales de quienes depende la manera como la empresa se relaciona con los demás agentes del entorno. 


\subsection{Fuentes de innovación}

Drucker (1998), fue uno de los pioneros en establecer cuáles eran las fuentes de innovación de las organizaciones en su intento por sistematizar la administración de la innovación, planteando siete fuentes de innovación distribuidas por fuera de la empresa y dentro de ella, pues atribuye la falta de disciplina en ella al no tener conciencia de hacia dónde dirigir sus esfuerzos para iniciar el proceso de innovación. Los considera como una serie de fuentes que pueden dar origen a un proceso de innovación, y no se restringe a las actividades de investigación y desarrollo (Formichella, 2005).

Dentro de los diferentes tipos de fuentes, Drucker (1998), define las fuentes de innovación por fuera de la organización como el conjunto de cambios demográficos, cambios de percepción y nuevos conocimientos. Dentro de la empresa, las fuentes más importantes anotan los sucesos no esperados, las incongruencias, necesidades de los procesos y cambios en el mercado e industria.

Con más detalle, Formichella (2005), resalta en su estudio del trabajo de Drucker sobre las fuentes de innovación, que el nuevo conocimiento está marcado por un periodo largo de transformación para la convergencia del conocimiento, es decir, el llevar aquel conocimiento disponible hacia una aplicación concreta. Este nuevo conocimiento está fuertemente ligado con los cambios externos que urgen en el entorno social, filosófico, político e intelectual, que entregan una nueva percepción, significado y manera de comprender las ideas o base del conocimiento disponible.

Los cambios demográficos, asociados a las modificaciones de variables tales como cambios de tamaño, grupos de edad, composición, trabajo, nivel de educación y de ingresos, los cuales, de manera individual, pueden comportarse como apalancadores de la actividad creativa y desencadenar un nuevo proceso de innovación.

Con respecto a las fuentes internas de la organización, Drucker (1998), resalta inicialmente el factor inesperado, el cual puede estar asociado con un evento exitoso, fracaso u otro acontecimiento. Para sacar provecho de esta fuente, el mismo autor destaca una alta capacidad del empresario para «indagar sobre lo que es necesario cambiar en la organización para asimilar lo que está ocurriendo y obtener de ello un beneficio».

El factor incongruencia es otra fuente interna de innovación y está relacionada con no tratar de comprender las causas del no funcionamiento ideal de un procedimiento, producto u evento, sino más bien, intentar en convertir esa incongruencia en una oportunidad (Formichella, 2005).

Finalmente, la necesidad del proceso es un factor que resalta, más que aprovechar las eventualidades fortuitas de lo inesperado e incongruente, apegarse a la línea trazada de trabajo. Las fuentes de innovación, identificadas por Drucker (1998) en su trabajo, son un listado conservador y útil no solo para enfocar el proceso de innovación, sino

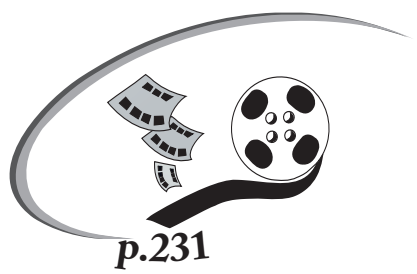
que además indica, de manera explícita, que no todas las innovaciones surgen de ideas brillantes. Estas grandesideas, porsu vaguedad y grado de generalización, muchas veces no son implementadas y caen en el olvido. Por su parte, propone prestar especial cuidado al listado de fuentes expuestas anteriormente como conducta racional, ya que las variables relacionadas tienen un impacto significativo 
dentro de la organización, pues su estudio y análisis detallado indican la manera en que esta concentrará sus esfuerzos para construir su oferta diferenciadora o propuesta de valor.

\subsection{Modelos de innovación}

Las organizaciones empresariales, ante la necesidad de aplicar procesos efectivos de innovación y tecnología, han originado una amplia diversificación de comportamientos con los que algunos autores han planteado modelos de innovación empresarial que tratan de explicar la dinámica innovadora, sus elementos e interacciones de las organizaciones. Dichos modelos, de ninguna manera buscan ser rígidos o absolutos; por el contrario, tratan de proporcionar cierta flexibilidad dependiendo del tipo de sistema empresarial.

\subsubsection{Modelo lineal}

Este modelo está caracterizado por la dependencia casi total con la investigación básica, con una carencia de retroalimentación efectiva entre sus componentes y con un comportamiento demasiado consecuente y poco realista entre sus elementos. Sin embargo, su aplicabilidad es muy útil en las organizaciones, donde la tecnología está basada en el desarrollo científico interno y en una fuerte capacidad de recursos, la industria farmacéutica, la industria de biotecnología y las dedicadas a la creación o desplazamiento de nuevas materias primas.

\subsubsection{Modelo interactivo o Kline}

Como su nombre lo indica, es un modelo que captura la interacción y retroalimentación de información existente entre sus elementos. Una de sus características más importantes es que no limita su operatividad a la dependencia de alguno de sus elementos, sino que el proceso de innovación puede iniciarse indistintamente en la investigación básica, en el diseño, en la comercialización o en cualquiera de sus componentes, lo cual deja de centrar el proceso solamente a la ciencia y le ofrece un papel protagónico a la organización empresarial. Este modelo, demuestra que la ciencia no solamente está ceñida a crear, sino también a transformar. Por lo general, es más flexible y aplicable a cualquier tipo de empresa, ya que se puede ajustar a sus procesos productivos y a sus necesidades.

En el mundo empresarial, en la última década, se ha fomentado, dentro de los líderes organizacionales, una nueva mentalidad mucho más abierta y colaborativa para desarrollar sus procesos, en la que para ser competitivo hay que tener en cuenta otros factores, como la necesidad de ofrecer soluciones con un alto valor agregado, la revolución de Internet y las redes sociales, así como la adaptación de diferentes soluciones para distintos

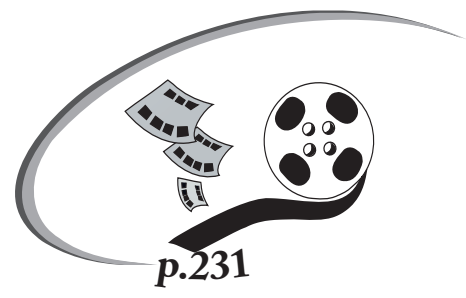
entornos. Es decir, la innovación es abierta y ya no es cuestión de ofrecer una única respuesta a un determinado desafío, sino que varía en función del ambiente en el que se encuentra la empresa, los aliados con los que cuente y las relaciones con el entorno (Bernardo, 2014). 


\subsubsection{El modelo de innovación organizacional}

Según Daft (2005, p. 404), este modelo se define como «la adopción de una idea o conducta nueva en la industria, mercado o ambiente en general de la organización».

Así mismo, el modelo de innovación organizacional se traduce en el acogimiento de ideas o comportamientos novedosos en las empresas, lo cual posibilita dentro de su ambiente, el desarrollo o gestación de ideas en la organización que modifican el entorno organizacional de los trabajadores en lo que se refiere a la forma de trabajo de los empleados, el impacto en su cultura organizacional, además, la agilización de los procesos que denotan una ventaja positiva en el cumplimiento de tiempos, así como la contribución a la eficiencia y la eficacia de la organización (Yoguel y Boscherini, 1996).

Es decir, los procesos de innovación organizacional se tornan importantes siempre y cuando su gestión se ancle con la estrategia empresarial, pues si no lo está se tornará difícil el manejo de este tipo de modelo; así mismo, el conjunto de cambios que reúne, intenta agrupar actividades que anteriormente se encontraban aisladas y que en dicho punto de la gestión de los procesos organizativos podrían pasar inadvertidas o encontrarse no alineadas conforme a la gestión del cambio que en el momento compete.

En las organizaciones, hoy en día, la innovación forma una parte crucial para la obtención de ventajas en el mercado sobre los competidores; es aquella forma de interactuar de manera distinta en el mercado con el fin de ofrecer productos o servicios diferenciadores, en el caso descrito, hasta capacidades diferentes en el recurso humano de la empresa, por medio de la gestión de ambientes propicios para la gestación de ideas por parte de los empleados que coadyuven a la empresa, no solo a ofrecer bienes o servicios innovadores, sino a marcar una singularidad en el mercado, donde la competencia queda irrelevante frente a la implementación de nuevos procesos de la empresa.

\section{Conclusiones}

$\mathrm{L}^{\mathrm{s}}$ a correspondencia entre organizaciones $\mathcal{U}$ y su modelo de gestión permite la identificación del sistema de organización interna, así como acoplar el modelo de negocio que contempla la forma con la cual se crea valor para la organización; de igual forma, se evidencia cómo la innovación se ubica como un elemento estratégico que propicia, en la gestión de las organizaciones, la debida capacidad de reinventar las modalidades de operación y generar resultados dinámicos que contribuyen a la eficiencia administrativa en los debidos procesos de planeación, organización, dirección y control.

De acuerdo con la exploración, en la literatura pueden establecerse tres principales reflexiones: en primera instancia, las formas de hacer negocios en las organizaciones se han orientado, en gran parte, a la generación de valor agregado mediante la continua reinvención de los componentes del sistema, pero no a componentes específicos estructurales, sino puntualmente a factores clave del 
modelo, que permitan la creación de valor y la interactividad entre los actores que integran el sistema; en este sentido, puede afirmarse que la innovación está implícita en los momentos donde las organizaciones asumen y optan por reinventarse, ubicándose como una herramienta transversal que impac-ta sobre todos los elementos del modelo de negocio y de la gestión.

También, la innovación ha representado gran interés para el desarrollo empresarial e incluso de las naciones, ya que los modelos para su implantación han abarcado diferentes metodologías en el intento por generar el marco de conocimiento que permita a las organizaciones su apropiación y adaptación.
Como elemento agregado a este componente se destacaría la asociatividad del concepto a la investigación y el desarrollo científico y tecnológico, que facilita, la gestión de innovaciones de gran impacto.

Finalmente, puede concluirse que la innovación en los modelos de negocio facilitan el proceso de reinvención y cambio, que permiten la mayor generación de valor mediante la dinámica de componentes y formas que constituyen el modelo. Se requieren premisas y lecciones desde la perspectiva científica en aras de la integralidad de perspectiva en la arquitectura de los modelos de negocio en las organizaciones, y generar mayor impacto en sus diferentes componentes e integrantes. 


\section{Referencias bibliográficas}

Alvarado, L. y García, M. (2008). Características más relevantes del paradigma sociocrítico: su aplicación en investigaciones de educación. Sapiens 9(2) pp. 187-202.

Álvarez, C. (2009). Innovación, competitividad y nuevos modelos de negocio. Cuadernos de investigación EPG. pp. 1-15.

Amar, P. y Diazgranados, J. (2006). Modelo de relación estado-mercado para el fomento de la innovación. Investigación \& Desarrollo 14(1) pp. 200-225.

Amar, P.; Angulo, G.; Ortega, M. y Quintero, J. D. (2006). El sistema de innovación del departamento del Atlántico: una mirada desde la cibernética organizacional. Gerencia Tecnológica Informática 5(12) pp. 55-62.

Bernardo, A. (2014). ¿Qué modelos de innovación existen? Recuperado de: http:// blogthinkbig.com/modelos-de-innovacion/.

Casadesus-Masanell, R. y Ricart, J. E. (2007). Competing through business models. Consultado desde IESE Business School. Recuperado de: http://www.iese.edu/ research/pdfs/DI-0713-E.pdf

Daft, R. (2005). Teoría y diseño organizacional. México: Thomson.

Del Campo, A. (2009). Ventaja competitiva en tiempos de crisis. Revista líder empresarial. Recuperado de: http://www. liderempresarial.com/num173/7i.htm

Dorf, B. (Junio de 2015). Mentor roles, responsabilities and challenges. En J. Sandino (presidencia). Jornada de entre-namiento de Mentores AppsCo. Jornada llevada a cabo en Bogotá, Colombia.

Dorf, B. y Blank, S. (2012). The Startup Owner's Manual: The Step-by-step Guide for Building a Great Company. California: K\&S Ranch Inc.

Drucker, P. (1998). The discipline of innovation. Harvard Business Review. 63(3) pp. 67-72.

Drucker, P. (2007). The practice of management. UK: Routledge.

Etzkowitz, H. \& Leydesdorff, L. (2000). The dynamics of innovation: from National Systems and "Mode 2" to a Triple Helix of university-industry-government relations. Research Policy (29) pp. 109-123.

Formichella, M. (2005). La evolución del concepto de innovación y su relación con el desarrollo. Recuperado: http:// www.fidamerica.cl/admin/docdescargas/ centrodoc/centrodoc_1142.pdf

Foster, R. (1987). Innovación: la estrategia del triunfo. Barcelona: Editorial Folio.

Freeman, C. (1975). La teoría económica de la innovación industrial. Madrid: Alianza.

Gee, S. (1981). Technology transfer, innovation and international competitiveness. Nueva York: Wiley \& Sons.

Goldratt, E. \& Cox, J. (2010). La meta, un proceso de mejora continua. Buenos Aires: Editorial Granica. 
Hamel, G. (2006). The why, what, and how of management innovation. Harvard Business Review, pp. 1-11.

Jofré, E. (2002). Modelo de diseño y ejecución de estrategias de negocio. Serie gestión. (35), pp. 1-43.

Johnson, M., Christensen, C. \& Kagermann, H. (2008). Reinventing your business model. Harvard Business Review. 86 (12), pp. 51-59.

López, O. (2014). Creando una empresa de éxito: Claves para pasar de la idea a la empresa. Create Space.

Magretta, J. (2002). Why business models matter. Harvard Business Review. Recuperado de: https://hbr.org/2002/05/whybusiness-models-matter.

Márquez, J. F. (2010). Innovación en modelos de negocio: la metodología de Osterwalder en la práctica. MBA EAFIT. (1), pp. 30-47.

Melamed, E. (2015). Procesos administrativos en la fundamentación teórica de la gerencia del servicio al cliente. Revista FACCEA 5(2), pp. 118-131.

Mendoza, J. M. (2000). Innovación por lo alto: imaginación y acción en la empresa. Bogotá: Asesores del 2000.

Mendoza, J. M. (2005). Seis sigmas: Hacia la cumbre de la calidad. Pensamiento \& gestión. (19), pp. 101-117.

Montoya, A.; Montoya, I. y Castellanos, O. (2010). Situación de la competitividad de las pyme en Colombia: elementos actuales y retos. Agronomía colombiana. 28 (1), pp. 107-117.
OCDE (2005). Manual de Oslo, directrices para la recogida e interpretación de información.

Ortiz, L. E. (2014). Análisis del sistema regional de ciencia, tecnología e innovación en el departamento del Atlántico-Colombia. Desarrollo gerencial. 4 (2), pp. 72-103.

Osterwalder, A. (2007). How to describe and improve your Business Model to Compete Better. Melbourne: La Trobe University.

Osterwalder, A. y Pigneur, Y. (2013). Generación de modelos de negocio. España: Grupo Planeta.

Peña, C., Soria, M. y Velázquez, L. (2012). Gestión del cambio e innovación organizacional como factor de la responsabilidad social. Revista Iberoamericana para la Investigación y el Desarrollo Educativo.

Porter, M. (2009). Ser competitivo. Barcelona: Deusto.

Porter, M. (1991). The competitive advantage of nations. Nueva York: Free Press.

Revilla, E. (1999). Organización que aprende hacia la gestión del conocimiento. Recuperado de: www.fcee.ulpgc.es/ Acede98/ acede/mesa02/2_04c.htm

Schumpeter, J. A. (2010). Capitalism, socialism and democracy. New York: Harper y Brothers.

Schumpeter, J. A. (1989). Economic theory and entrepreneurial history. Cambridge: Harvard University Press.

Stiglitz, J. (2002). El malestar en la globalización. Buenos Aires: Santillana. 
Tejada. A, (2003). Los modelos actuales de gestión en las organizaciones. Gestión del talento, gestión del conocimiento y gestión por competencias. Psicología desde el Caribe. (12), pp. 115-133.

Turner, S. \& DeVaughn, S. (2008). Net Value: How to Develop a Winning Value Proposition for Marketing in the Digital Culture. San Francisco: Turner DeVaughn Network.

Wilmot, W. \& Carlson, C. (2006). Innovation: the five disciplines for creating what customers want. New York: Crown Publishing Group.
Yoguel, G. \& Boscherini, F. (1996). La capacidad innovativa y el fortalecimiento de la competitividad de las firmas. El caso de las pymes exportadoras argentinas. Recuperado de: http://goo.gl/47JRSr

Zaltman, G., Duncan, R. \& Holbek, J. (1973). Innovations and Organizations. New York: Wiley Editions.

Zott, C. \& Amit, R. (2005). Business model design and the performance of entrepreneurial firms. INSEAD: Wharton University of Pennsylvania. 
